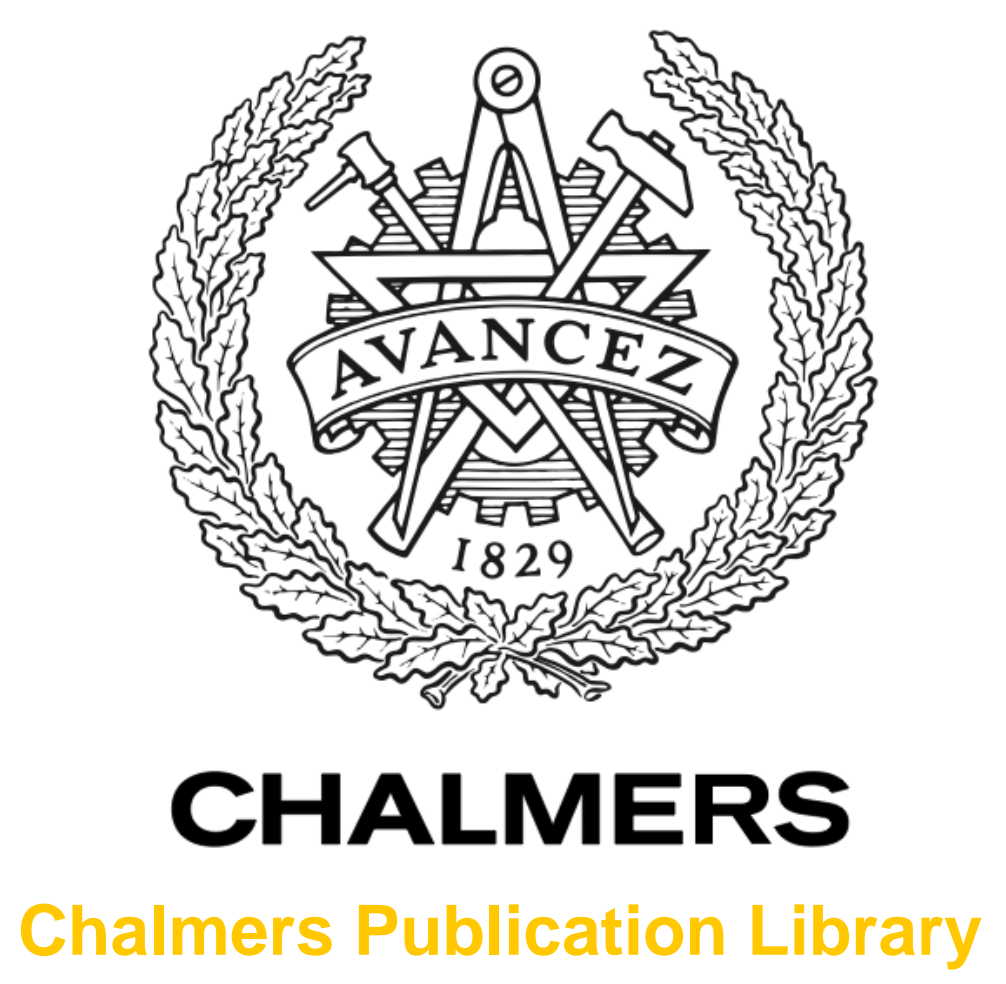

\title{
Dispersion Characteristics of a Metamaterial-Based Parallel-Plate Ridge Gap Waveguide Realized by Bed of Nails
}

This document has been downloaded from Chalmers Publication Library (CPL). It is the author's version of a work that was accepted for publication in:

IEEE Transactions on Antennas and Propagation (ISSN: 0018-926X)

Citation for the published paper:

Polemi, A. ; Maci, S. ; Kildal, P. (2011) "Dispersion Characteristics of a Metamaterial-Based Parallel-Plate Ridge Gap Waveguide Realized by Bed of Nails". IEEE Transactions on Antennas and Propagation, vol. 59(3), pp. 904-913.

http://dx.doi.org/10.1109/tap.2010.2103006

Downloaded from: http://publications.lib.chalmers.se/publication/138801

Notice: Changes introduced as a result of publishing processes such as copy-editing and formatting may not be reflected in this document. For a definitive version of this work, please refer to the published source. Please note that access to the published version might require a subscription. 


\title{
Dispersion Characteristics of a Metamaterial-Based Parallel-Plate Ridge Gap Waveguide Realized by Bed of Nails
}

\author{
A. Polemi, Member, IEEE, S. Maci, Fellow, IEEE, P-S. Kildal, Fellow, IEEE
}

\begin{abstract}
The newly introduced parallel-plate ridge gap waveguide consists of a metal ridge in a metamaterial surface, covered by a metallic plate at a small height above it. The gap waveguide is simple to manufacture, especially at millimetre and sub-millimetre wave frequencies. The metamaterial surface is designed to provide a frequency band where normal global parallel-plate modes are in cut-off, thereby allowing a confined gap wave to propagate along the ridge. This paper presents an approximate analytical solution for this confined quasi-TEM dominant mode of the ridge gap waveguide, when the metamaterial surface is an artificial magnetic conductor in the form of a bed of nails. The modal solution is found by dividing the field problem in three regions, the central region above the ridge and the two surrounding side regions above the nails. The fields within the side regions are expressed in terms of two evanescent TE and TM modes obtained by treating the bed of nails as an isotropic impedance surface, and the field in the central ridge region is expanded as a fundamental TEM parallel-plate mode with unknown longitudinal propagation constant. The field solutions are linked together by equalizing longitudinal propagation constants and imposing point-continuity of fields across the region interfaces, resulting in a transcendental dispersion equation. This is solved and presented in a dispersion diagram, showing good agreement with a numerical solution using a general electromagnetic solver. Both the lower and upper cut-off frequencies of the normal global parallel-plate modes are predicted, as well as the quasi-TEM nature of the gap mode between these frequencies. The evanescent fields in the two side regions decay very rapidly away from the ridge, being in the order of $100 \mathrm{~d} B$ per lower cut-off wavelengths over most of the single-mode bandwidth.
\end{abstract} face

Keywords - bed of nails, waveguide, metamaterials, EBG, artificial sur-

\section{INTRODUCTION}

$\mathrm{T}$ H IS paper deals with the modal analysis of a new typology of metamaterial-based waveguide, particularly suitable for millimetre and sub-millimetre realizations [1] [2]. The basic structure is shown in Fig. 1. In the above-mentioned range of frequencies, this solution presents advantages compared to existing technologies like hollow rectangular waveguide (HRW) and microstrip lines. Indeed, HRW can be manufactured in two parts and joined together, but not without consequent problems of good electrical contacts. On the other hand, microstrip lines suffer from losses with increasing frequency, limited power handling capability, and spurious resonances when encapsulated. Other solutions like Substrate Integrated Waveguide (SIW) [3] exhibit undesired losses due to substrate at increasing frequencies. Therefore, there is still needs to find new technological solutions for waveguides above $30 \mathrm{GHz}$ that have low losses and are cheap to manufacture. The structure analyzed in this paper is shown in Fig. 1 and can be seen as a particular real-

A. Polemi is with Department of Information Engineering, University of Modena and Reggio Emilia, Via Vignolese 905, 41100 Modena, Italy (alessia.polemi@unimore.it). S. Maci is with Department of Information Engineering, University of Siena, Via Roma 56, 41100 Modena, Italy (macis@dii.unisi.it). P-S. Kildal is with 3Department of Signals and Systems, Chalmers University of Technology, Gotheborg, Sweden (per-simon.kildalechalmers.se) ization of the more general ridge gap waveguide introduced in [1] [2]. It is composed of a pin surface, so called "fakir's bed" or "bed of nails", surrounding a metallic ridge, and everything covered by a metal plate. The bed of nails can be immersed in dielectric substrate to reduce the size and the following analysis will account for it. However, in applications at millimetre wave frequency, the better solution is avoiding any dielectric. The fakirs bed is designed in such a way to create an artificial magnetic surface [4], over a certain frequency range. With the metallic top cover, it forms a PEC-PMC parallel plate waveguide which will have a cut-off for all modes when the gap height $h$ is smaller than a quarter of a wavelength $(\mathrm{PEC}=$ Perfect Electric Conductor, PMC = Perfect Magnetic Conductor). This implies that a quasi-TEM mode can propagate in the gap along the ridge without spreading into the cut-off region above the pin surface. The large bandwidth of the high impedance of the pin surface allows a quasi-TEM mode propagation over a large frequency band without significant dispersion.

The ridge gap waveguide is a result of research on so called soft and hard surfaces [5], which in their simplest form are realized by corrugations. They can in the ideal form be represented by a PEC/PMC strip grid, as explained in [6]. A hard surface covered by a PEC was in [7] and [8] shown to suppress higher order parallel plate modes. Instead there exist several degenerate local quasi-TEM waves following the ridges, as studied in detail by numerical simulations in [9]. Related surface waves in open hard structures were detected already in [12] but these follow individual grooves of corrugated surfaces rather than individual ridges, which is the case for the gap waveguides. The new ridge waveguide is a direct consequence of the local waves studied numerically in [9]. The present paper represents the first attempt to find an analytic solution to the modal fields. The gap waveguide can also be understood as a miniaturized hard waveguide having two PEC walls and two PMC walls [10], where the vertical PMC walls of the present ridge gap waveguide are realized by the cut-off between two parallel plates which gives a very wideband solution compared to the narrow-band dielectric slab and FSS wall realizations in [10] and [11], respectively.

The main purpose of the present paper is to model the dispersion characteristics of the quasi-TEM mode in an analytical form, in order to take under control the dispersion effects and to design the structure in the appropriate frequency band. The fields associated with this mode are mainly transverse to the direction of propagation, but small longitudinal components of the fields are also anticipated and included in the formulation in Section II. The field is expected to have an exponential decay laterally away from the ridge into the region above the pins. In the 
present formulation, we will not describe the field inside the bed of nails itself, but instead represent the latter by a spatially dispersive anisotropic homogeneous medium characterized by an equivalent homogeneous reflection coefficient according to [4]. The assumed field expressions in the region above the ridge and the two side regions above the surrounding pin surfaces are used to establish point matching continuity at the interfaces between the regions, and thereby to obtain a dispersion equation.

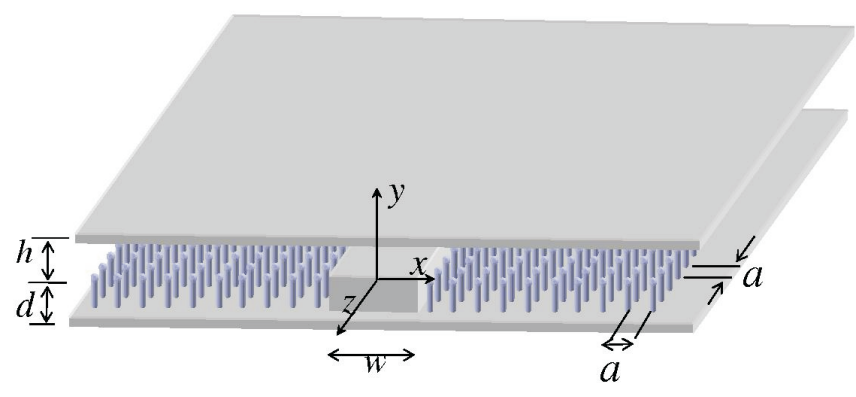

Fig. 1. Geometry of the ridge gap waveguide embedded in a bed of nails

\section{BED OF NAILS PHENOMENOLOGY}

As a fist step we analyze the phenomenology associated with the bed of nails shown in Fig. 2. The nails are constituted by small metallic cylinders of height $d$, with radius $b$, and spacing $a$ in both $x$ and $y$ directions. For completeness, we assume the nails to be embedded in a host medium with permittivity $\varepsilon_{h}$; in practical application however, it is better to have nails immersed in free space. In [4] the same geometry has been studied by assuming a plane wave with wave vector $\bar{k}_{i}$ incident on the textured surface. Following [4], the bed of nails is regarded as a
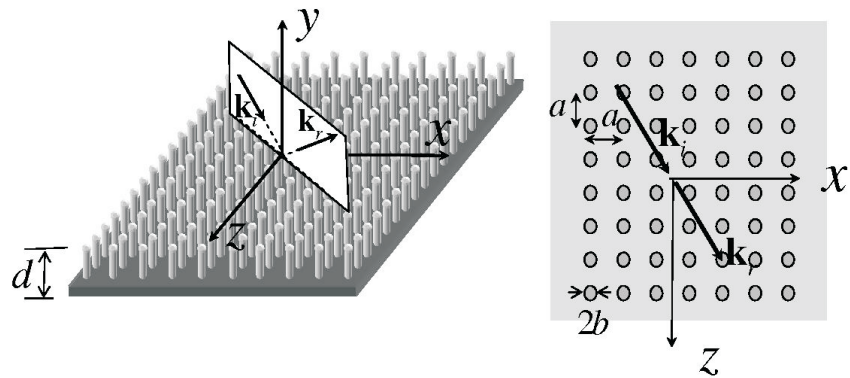

Fig. 2. Bed of nails basic geometry. (left) 3D-view; (right) top-view.

spatial and frequency dispersive medium whose permittivity is characterized by the tensor

$$
\underline{\underline{\varepsilon}}\left(\omega, k_{y}\right)=\varepsilon_{0} \varepsilon_{h}\left(\hat{x} \hat{x}+\hat{z} \hat{z}+\varepsilon_{y y}\left(\omega, k_{y}\right) \hat{y} \hat{y}\right) .
$$

In (1),

$$
\varepsilon_{y y}\left(\omega, k_{y}\right)=1-k_{p}^{2} / k_{h}^{2}-k_{y}^{2}
$$

where $k_{h}=\sqrt{\varepsilon_{h}} k$ is the wave number of the host medium and

$$
k_{p}=\frac{1}{a} \sqrt{\frac{2 \pi}{\ln \left(\frac{a}{2 \pi b}\right)+0.5275}}
$$

is the plasma wave number accounting for the local spatial dispersion [13], only dependent on the geometrical lattice properties. The mathematical model described by (1) arises from an evolution for the wire medium model, which accounts for spatial dispersion. In [4] it is shown that the homogenized medium can support three different modal solutions: a transverse electromagnetic (TEM) mode, a transverse magnetic (TM- $y$ ) mode, and a transverse electric (TE- $y$ ) mode. Imposing the continuity of all the possible fields at the air-medium interface (including vertical component of induced fields), allows one to calculate the reflection coefficients. For an impinging plane TM wave [4] we obtain

$$
\begin{array}{r}
\Gamma^{T M}\left(k_{y}\right)= \\
-\frac{k_{h} k_{p}^{2} \tan \left(k_{h} d\right)-k_{\|}^{2} \gamma_{T M} \tanh \left(\gamma_{T M} d\right)+\varepsilon_{h} \gamma_{0}\left(k_{p}^{2}+k_{\|}^{2}\right)}{k_{h} k_{p}^{2} \tan \left(k_{h} d\right)-k_{\|}^{2} \gamma_{T M} \tanh \left(\gamma_{T M} d\right)-\varepsilon_{h} \gamma_{0}\left(k_{p}^{2}+k_{\|}^{2}\right)}
\end{array}
$$

where $k_{\|}=\sqrt{k_{x}^{2}+k_{z}^{2}}=\sqrt{k^{2}-k_{y}^{2}}, \gamma_{0}=\sqrt{k_{\|}^{2}-k^{2}}$, and $\gamma_{T M}=\sqrt{k_{p}^{2}+k_{\|}^{2}-k^{2}}$. The reflection coefficient in (4) accounts for the penetration of TM mode inside the bed of nail through the attenuation constant gamma TM. This constant goes to infinity when the nails are densely packed. In (4), $k_{y}$ is dictated by the impinging direction of the plane wave on the surface. For a TE polarized incident plane wave, the reflection coefficient is that associated with a bare grounded slab, because the electric field, perpendicular to the surface of the pins, does not significantly interact with them. This is consistent with the assumption of very small radius; this assumption can be removed by using the approach in [14]; this will be subject of a future investigation. Within the hypothesis of TE not-perturbing nails, we obtain

$$
\Gamma^{T E}\left(k_{y}\right)=-\frac{\sqrt{k_{h}^{2}-k_{\|}^{2}}-j \sqrt{k^{2}-k_{\|}^{2}} \tan \left(\sqrt{k_{h}^{2}-k_{\|}^{2}} d\right)}{\sqrt{k_{h}^{2}+k_{\|}^{2}}-j \sqrt{k^{2}-k_{\|}^{2}} \tan \left(\sqrt{k_{h}^{2}-k_{\|}^{2}} d\right)} .
$$

In (4) and (5) we have maintained the definition in [4], where the reflection coefficients are are those associated with the magnetic field; thus, when $d=\lambda_{h} / 4$ we have $\Gamma^{T M(T E)}=-1(+1)$. Finally, the validity of this coefficient is given in [4] for values of $k_{h}$ a comparable with $\pi$.

As mentioned above, when the metallic pins are closely packed, which means $a / d<<1$ [15], one has $\gamma_{T M} \rightarrow k_{p} \rightarrow 1 / a \rightarrow \infty$ (while $b / a$ is kept constant), and

$$
\Gamma^{T M}\left(k_{y}\right) \simeq \Gamma=-\frac{k_{h} \tan \left(k_{h} d\right)+\varepsilon_{h} \gamma_{0}}{k_{h} \tan \left(k_{h} d\right)-\varepsilon_{h} \gamma_{0}}
$$

which is the solution of the reflection problem when only the TEM mode is considered, as expected. Thus, when the pins are densely packed, the wire medium behaves approximately as an anisotropic material with $\varepsilon_{x x}=\varepsilon_{z z}=\varepsilon_{h}$ and $\varepsilon_{y y}=\infty$. Its equivalent impedance is

$$
Z_{s}=j \frac{\xi}{\sqrt{\varepsilon_{h}}} \tan \left(k_{h} d\right)
$$

and it behaves as an inductance when (i) $d \leq \lambda_{h} / 4$, and as a capacitance when (ii) $\lambda_{h} / 4<d \leq \lambda_{h} / 2$. When $d=\lambda_{h} / 4$, the 
equivalent impedance represents a PMC. The same condition as in [15] is in [16] referred to as an asymptotic boundary condition when dealing with corrugations and strip grids. Therefore, (7) represents also an asymptotic boundary condition of a bed of metal nails.

\section{DISPERSION EQUATION FOR PEC-PLATE COVERED BED OF NAILS}

The guiding phenomenon on the bed of nails surface is strongly modified by the presence of a metallic plate. They form together a parallel plate waveguide where one face is PEC and the other is reactive through the homogenized surface described in Section II (see Fig. 3). For simplicity, hereinafter we consider free-space embedded nails $\left(\varepsilon_{h}=1\right)$. The same

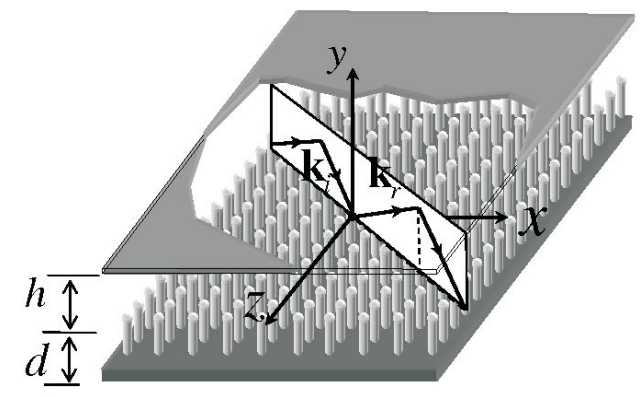

Fig. 3. Reflection phenomenology supported by the bed of nails surface covered by a PEC plate.

has been done in [17], but one of the parallel-plate faces was there constituted by an anisotropic hard/soft surface along the direction of propagation, obtained as a periodic structure made up of corrugations. Here, the fakir's bed homogenized surface is completely $3 \mathrm{D}$, and then the modal field configuration supported is more complicated. The wave bouncing in the gap between the two faces can still be described by the reflection coefficients $\Gamma^{T M}\left(k_{y}\right)$ and $\Gamma^{T E}\left(k_{y}\right)$, where now the $k_{y}$ value is the solution of the eigenvalue problem obtained by imposing the vanishing of the tangential electric field at the upper wall, i.e. $\left[e^{j k_{y} y}+\Gamma^{T M, T E}\left(k_{y}\right) e^{-j k_{y} y}\right]_{y=h}=0$. In absence of the dielectric background in the bed of nails, TE mode solution is that associated with the resonance between the upper and lower PEC walls, i.e. $k_{y}=\pi /(h+d)$. For TM modes, the equation to be solved can be conveniently rewritten by employing (4), which leads to

$$
\begin{aligned}
& \frac{k_{y}}{k} \tan \left(k_{y} h\right)+\left[1-\frac{k^{2}-k_{y}^{2}}{k_{p}^{2}+k^{2}-k_{y}^{2}}\right] \tan (k d)+ \\
& +\frac{k^{2}-k_{y}^{2}}{k_{p}^{2}+k^{2}-k_{y}^{2}} \frac{\sqrt{k_{p}^{2}-k_{y}^{2}}}{k} \tan \left(\sqrt{k_{p}^{2}-k_{y}^{2}} d\right)=0
\end{aligned}
$$

The above equation can be easily interpreted as a circuit series resonance equation of the kind

$$
Z_{0, T M}^{s c}\left(k_{y}\right)+\left[1-\eta\left(k_{y}\right)\right] Z_{T E M}^{s c}+\eta\left(k_{y}\right) Z_{T M}^{s c}\left(k_{y}\right)=0
$$

where, referring to the equivalent circuit in Fig. $4, Z_{0, T M}^{s c}\left(k_{y}\right)=$ $j \xi \frac{k_{y}}{k} \tan \left(k_{y} h\right)$ is the TM modal impedance seen at the pin surface towards the PEC cover plate, while $Z_{T E M}^{s c}=j \xi \tan (k d)$ and $Z_{T M}^{s c}\left(k_{y}\right)=j \xi \frac{\sqrt{k_{p}^{2}-k_{y}^{2}}}{k} \tan \left(\sqrt{k_{p}^{2}-k_{y}^{2}} d\right)$ are the TEM and TM modal impedances seen from the nail's top surface towards the short circuited nail's medium, respectively (note that the TM mode feels the pins with a characteristic dispersive impedance $\left.\xi \frac{\sqrt{k_{p}^{2}-k_{y}^{2}}}{k}\right)$. In (9), $\eta\left(k_{y}\right)=\frac{k^{2}-k_{y}^{2}}{k_{p}^{2}+k^{2}-k_{y}^{2}}$ is a parameter

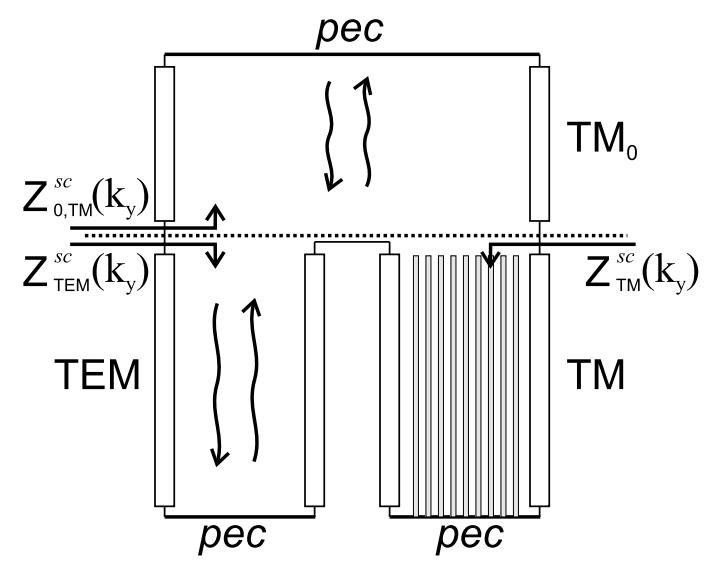

Fig. 4. Equivalent resonant circuit associated with the dispersion equation of the bed of nails surface covered by a top metal plate at height $h$.

which weights the TM mode relative to the TEM mode inside the bed of nails, and it can be easily derived from the continuity of the magnetic field at interface, or equivalently of the current in a series resonant circuit. In particular, when $k_{p}^{2}>>k^{2}-k_{y}^{2}$, corresponding to the densely packed bed of nails, $\eta\left(k_{y}\right) \rightarrow 0$; thus, the resonance condition becomes

$$
Z_{0, T M}^{s c}\left(k_{y}\right)+Z_{T E M}^{s c}=0
$$

thus leading to the conventional dispersion equation where the TEM mode is the only one considered inside the bed of nails. Equation (8), or possibly (10) can be solved numerically. Here, the intrinsic Matlab FSOLVE routine is employed, which finds a root of a system of nonlinear equations, given a certain starting point [18]. Results in terms of frequency are shown in Fig. 5 for a reference geometry (see Fig. 3) with $h=1 \mathrm{~mm}$ (height of gap), $d=7.5 \mathrm{~mm}$ (height of pins), $a=2 \mathrm{~mm}$ (periodicity of pins) and $b=0.5 \mathrm{~mm}$ (radius of pins). Eigenvalues $k_{y}$ of the complete TM resonance equation (8) and of the approximated TEM resonance equation (10) are reported as solid and dashed lines, respectively. The two solutions agree well, especially in the low frequency regime, where the bed of nails appears more closely packed with respect to the wavelength, and then the surface acts like the surface impedance in (7). In Fig. 5, the TE $k_{y}$ eigenvalue is also shown (dashed line), which is constant in frequency and dictated by the PEC-PMC resonance, i.e. $k_{y}=\pi /(h+d)$. As the frequency increases, the two solutions slightly tend to veer. Let us discuss the type of wave in the various frequency region. For $f<f_{(d=\lambda / 4)}$, $k_{y}$ is found to be purely imaginary (see Fig. 6); there, a TM surface wave propagate along the interface without being substantially affected by the top cover. In the frequency region between the two resonance frequencies $f_{(d=\lambda / 4)}$ and $f_{(d=\lambda / 2)}$, $k_{y}$ is real and a bouncing wave is standing in the gap as depicted in Fig. 3. In this range, we identify two regions; the first 


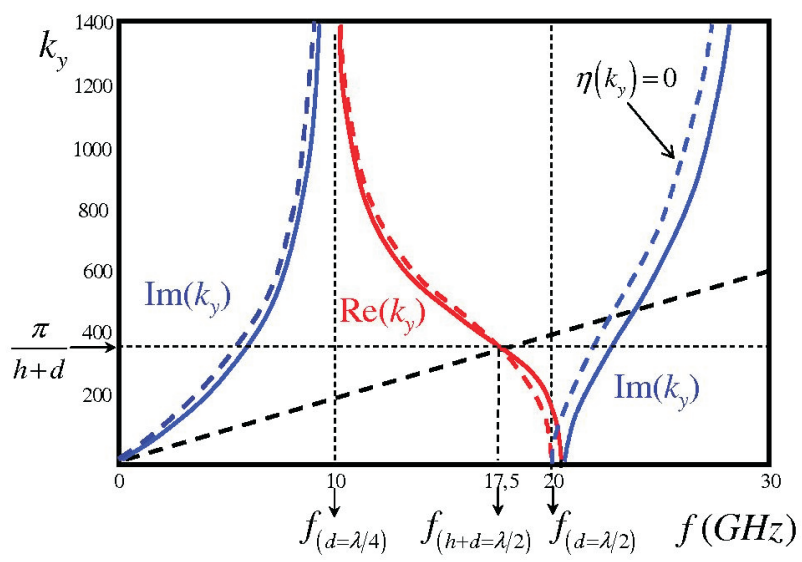

Fig. 5. TM dispersion equation of the complete (8) form (solid lines) and approximated (10) (dashed lines). The eigenvalue solution is real or imaginary in three frequency regions, separated by vertical dashed lines. TE eigenvalue is also shown (constant short-dashed line). The light line is also depicted (long dashed line). These results have been obtained for a reference geometry (see Fig. 3) with $h=1 \mathrm{~mm}$ (height of gap), $d=7.5 \mathrm{~mm}$ (height of pins), $a=2 \mathrm{~mm}$ (periodicity of pins) and $b=0.5 \mathrm{~mm}$ (radius of pins).

$\left(f_{(d=\lambda / 4)} ; f_{(d+h=\lambda / 2)}\right)$, where $k_{y}$ is real and greater than $k$, that implies attenuation along any direction along the surface, and the second region $\left(f_{(d+h=\lambda / 2)} ; f_{(d=\lambda / 2)}\right)$ where the propagation is admitted along the surface. The first range is the stop band of the structure, whose upper bound is the cut-off frequency of the first TE mode. The upper cut-off of the stop band, corresponds to the lower cut-off frequency. In order to better identify the stop band, a dispersion diagram $f$ versus $k_{z}$ is shown in Fig. 6 where $k_{z}=\sqrt{k^{2}-k_{y}^{2}}$, with $k_{y}=\widetilde{k}_{y}$ for TM solution (dotted line), and $k_{y}=\widetilde{\widetilde{k}}_{y}$ for TE solution (dashed line). The light line

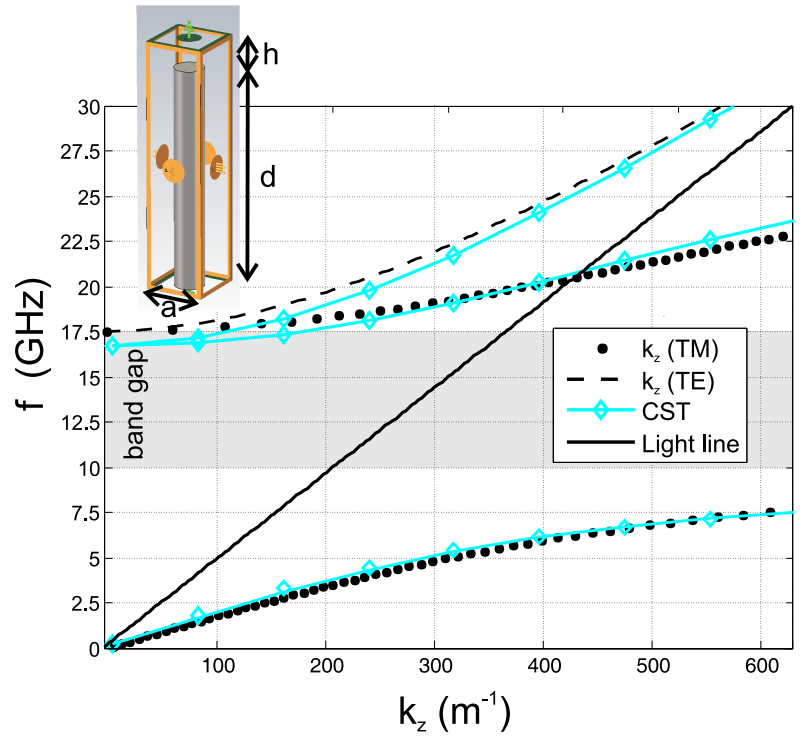

Fig. 6. Frequency versus real part of $k_{z}$ for TM (black dots) and TE (dashed line) solutions of the pertinent eigenvalues problems. The light line is also shown (solid line). These results have been obtained for the same geometrical parameters used for Fig. 5 .

is shown. From the dispersion diagram in Fig. 6 it is possible to observe the stop band (shadowed area) where no propagation is permitted. As expected, there is a surface wave propagating at the low frequency up to $10 G H z$, corresponding to $f @_{(d=\lambda / 4)}$. The dispersion diagram is in the same graph compared with results from a commercial software (CST Microwave Studio, diamond line), where the basic cell of the periodic structure is depicted in the inset of Fig. 6, and phase shifts along $x$ and $z$ directions have been imposed. The agreement is found to be overall good.

\section{DISPERSION EQUATION FOR A BED OF NAILS-BASED RIDGE GAP WAVEGUIDE}

We can realize a gap waveguide by inserting a conducting ridge of width $w$ into the bed of nails structure in the geometry in Fig. 3, thus leading to the structure in Fig. 1. From an intuitive point of view, we expect that the fundamental modal field will be propagating along the ridge, with a mechanism very similar to that of the narrow standard parallel-plate waveguide, with a quasi-TEM behavior. This mode will match the evanescent modes supported by the surrounding cut-off structure, in which we assume that only the first TM and TE modes (with respect to $y$ ) will be present. In particular, in order to achieve the field confinement in the ridge region, the cut-off modes must decay laterally away from the ridge (along direction $x$ ). In order to prove this approximation, we have run a simulation in CST MicrowaveStudio in order to get the transverse field distribution. This result is plotted for a few frequencies in Fig. 7, where the amplitude of the vertical electric field is shown. It is easy to recognize that the bed of nails surface stops propagation along the lateral direction and keeps the desired confinement of the field in the ridge region for frequencies within the stop band.

Under the approximations used above, we can now write the expressions for the fields. In particular, the TMy evanescent mode fields in the region above the bed of nails are,

$$
\begin{gathered}
H_{x}=j A_{T M} \frac{k_{z}}{\sqrt{k^{2}-\widetilde{k}_{y}^{2}}} \widetilde{g}(x, z) \cos \left[\widetilde{k}_{y}(y-h)\right] \\
H_{z}=-A_{T M} \frac{\widetilde{\alpha}_{x}}{\sqrt{k^{2}-\widetilde{k}_{y}^{2}}} \widetilde{g}(x, z) \cos \left[\widetilde{k}_{y}(y-h)\right] \\
E_{x}=-j A_{T M} \frac{\xi}{k} \frac{\widetilde{\alpha}_{x} \widetilde{k}_{y}}{\sqrt{k^{2}-\widetilde{k}_{y}^{2}}} \widetilde{g}(x, z) \sin \left[\widetilde{k}_{y}(y-h)\right] \\
E_{y}=-j A_{T M} \frac{\xi}{k} \sqrt{k^{2}-\widetilde{k}_{y}^{2}} \widetilde{g}(x, z) \cos \left[\widetilde{k}_{y}(y-h)\right] \\
E_{z}=A_{T M} \frac{\xi}{k} \frac{k_{z} \widetilde{k}_{y}}{\sqrt{k^{2}-\widetilde{k}_{y}^{2}}} \widetilde{g}(x, z) \sin \left[\widetilde{k}_{y}(y-h)\right]
\end{gathered}
$$

where

$$
\widetilde{g}(x, z)=e^{-j k_{z} z} e^{-\widetilde{\alpha}_{x}(|x|-w / 2)} .
$$

The reference system is centered in the middle of the width $w$ of the ridge. In equations (11), $A_{T M}$ is an unknown coefficients while $\widetilde{k}_{y}$ is the eigenvalue solution of equation (8). Thus, the dispersion relation for this mode is $k^{2}=\left(-j \widetilde{\alpha}_{x}\right)^{2}+\widetilde{k}_{y}^{2}+k_{z}$, where the attenuation constant $\widetilde{\alpha}_{x}$ and the propagation constant 


\section{प्राIII IIIIII Vi|IIIIIIIIIII Frot

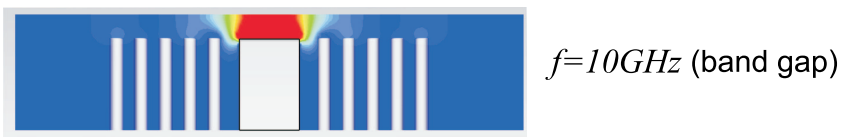 - IIIIT|IIIII

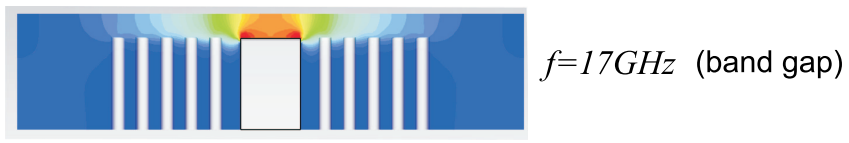 |}

Fig. 7. Normalized amplitude of the vertical (along $y$ ) electric field for different frequencies calculated through CST Microwave Studio

$k_{z}$ are also unknowns. A second $x$-evanescent TEy mode is assumed to be excited in the bed of nails region, whose expression is give by

$$
\begin{gathered}
E_{x}=j A_{T E} \frac{k_{z}}{\sqrt{k^{2}-\widetilde{\widetilde{k}}_{y}^{2}}} \widetilde{\widetilde{g}}(x, z) \sin \left[\widetilde{\widetilde{k}}_{y}(y-h)\right] \\
E_{z}=-A_{T E} \frac{\widetilde{\widetilde{\alpha}}_{z}}{\sqrt{k^{2}-\widetilde{\widetilde{k}}_{y}^{2}}} \widetilde{\widetilde{g}}(x, z) \sin \left[\widetilde{\widetilde{k}}_{y}(y-h)\right] \\
H_{x}=-j A_{T E} \frac{1}{\xi k} \frac{\widetilde{\widetilde{\alpha}}_{x} \widetilde{\widetilde{k}}_{y}}{\sqrt{k^{2}-\widetilde{\widetilde{k}}_{y}^{2}}} \widetilde{\widetilde{g}}(x, z) \cos \left[\widetilde{\widetilde{k}}_{y}(y-h)\right] \\
H_{y}=j A_{T E} \frac{1}{\xi k} \sqrt{k^{2}-\widetilde{\widetilde{k}}_{y}^{2}} \widetilde{\widetilde{g}}(x, z) \sin \left[\widetilde{\widetilde{k}}_{y}(y-h)\right] \\
H_{z}=A_{T E} \frac{1}{\xi k} \frac{k_{z}}{\sqrt{k^{2}-\widetilde{\widetilde{k}}_{y}^{2}}} \widetilde{\widetilde{g}}(x, z) \cos \left[\widetilde{\widetilde{k}}_{y}(y-h)\right]
\end{gathered}
$$

where

$$
\widetilde{\widetilde{g}}(x, z)=e^{-j k_{z} z} e^{-\widetilde{\widetilde{\alpha}}_{x}(|x|-w / 2)} .
$$

In (13), $A_{T E}$ is an unknown coefficient, while $\widetilde{\widetilde{k}}_{y}=\pi /(h+d)$ is the eigenvalue solution for the associated TE problem. The dispersion equation for this mode is $k^{2}=\left(-j \widetilde{\widetilde{\alpha}}_{x}\right)^{2}+\widetilde{\widetilde{k}}_{y}^{2}+k_{z}$, where the attenuation constant $\widetilde{\alpha}_{x}$ and the propagation constant $k_{z}$ are unknowns.

In the central region above the ridge, we assume a quasi-TEM mode, propagating along $z$ according to the phase factor $e^{-j k_{z} z}$, of the kind

$$
\begin{aligned}
& E_{y}=E_{0} \cos \left(\widehat{k}_{x} x\right) e^{-j k_{z} z} \\
& H_{x}=-E_{0} \frac{k_{z}}{\xi k} \cos \left(\widehat{k}_{x} x\right) e^{-j k_{z} z} \\
& H_{z}=-j E_{0} \frac{\widehat{k}_{x}}{\xi k} \sin \left(\widehat{k}_{x} x\right) e^{-j k_{z} z}
\end{aligned}
$$

where $E_{0}$ is a constant related to the incident power at the input port, and $\widehat{k}_{x}$ is a propagation constant along $x$, and $k^{2}=\widehat{k}_{x}^{2}+$ $k_{z}^{2}$. The overall unknowns are six, $A_{T M}, A_{T E}, \widetilde{\alpha}_{x}, \widetilde{\widetilde{\alpha}}_{x}, \widehat{k}_{x}, k_{z}$. Therefore, six equations are required to uniquely solve the problem. Three of these equations are provided by the dispersion relations in the three media, reported below for convenience:

$$
\begin{aligned}
k_{z}^{2} & =k^{2}-\widetilde{k}_{y}^{2}+\widetilde{\alpha}_{x}^{2} \\
k_{z}^{2} & =k^{2}-\widetilde{\widetilde{k}}_{y}^{2}+\widetilde{\widetilde{\alpha}}_{x}^{2} \\
k_{z}^{2} & =k^{2}-\widehat{k}_{x}^{2} .
\end{aligned}
$$

To find the remaining three equations, we enforce a razor blade continuity of the three field components across the separation walls $x= \pm w / 2$. The matched field components $E_{y}, H_{x}$ are namely all those belonging to the quasi-TEM mode. The nonmatched components $E_{x}, E_{z}$ and $H_{y}$ (only present in the bed of nails regions) are very weak, since the boundary conditions at the top cover impose vanishing of them. Thus, we obtain the three matching field equations

$$
\begin{array}{r}
-j A_{T M} \frac{\xi}{k} \sqrt{k^{2}-\widetilde{k}_{y}^{2}}=E_{0} \cos \left(\widehat{k}_{x} w / 2\right) \\
-A_{T M} \frac{\widetilde{\alpha}_{x}}{\sqrt{k^{2}-\widetilde{k}_{y}^{2}}}+A_{T E} \frac{1}{\xi k} \frac{k_{z} \widetilde{\widetilde{k}}_{y}}{\sqrt{k^{2}-\widetilde{\widetilde{k}}_{y}^{2}}}= \\
=-j E_{0} \frac{\widehat{k}_{x}}{k \xi} \sin \left(\widehat{k}_{x} w / 2\right) \\
j A_{T M} \frac{\tilde{k}_{z}}{\sqrt{k^{2}-\widetilde{k}_{y}^{2}}}-j A_{T E} \frac{1}{\xi k} \frac{\widetilde{\widetilde{\alpha}}_{y}}{\sqrt{k^{2}-\widetilde{\widetilde{k}}_{y}^{2}}}= \\
=-E_{0} \frac{k_{z}}{k \xi} \cos \left(\widehat{k}_{x} w / 2\right)
\end{array}
$$

that, added to the ones in (16), allow us to find the dispersion equation

$$
\begin{gathered}
\sqrt{k^{2}-k_{z}^{2}} \tan \left[\sqrt{k^{2}-k_{z}^{2}} \frac{w}{2}\right]\left(k^{2}-\widetilde{k}_{y}^{2}\right)+ \\
\frac{k_{z}^{2} \widetilde{k}_{y}^{2}-k^{2} \sqrt{k_{z}^{2}-k^{2}+\widetilde{k}_{y}^{2}} \sqrt{k_{z}^{2}-k^{2}+\widetilde{\widetilde{k}}_{y}^{2}}}{\sqrt{k_{z}^{2}-k^{2}+\widetilde{\widetilde{k}}_{y}^{2}}}=0
\end{gathered}
$$

that relates $k_{z}$ to frequency. 


\section{A. Numerical results}

The solution is found numerically and plotted as the dispersion diagram in Fig. 8, where the ridge width is $w=5 \mathrm{~mm}$. Again, the Matlab FSOLVE routine is employed [18]. The geometry is the same as that used in the previous results (see caption of Fig. 6). The curve has been successfully compared with the dispersion diagram obtained by a CST Microwave Studio simulation (diamond line). The CST reference structure is shown above the graph in Fig. 8. Our approximated solution tends to fail when the frequency is larger than the upper parallelplate cut-off frequency, because we have only included one fundamental mode for each region, and this is not sufficient. Higher order modes could be accounted for at higher frequencies.
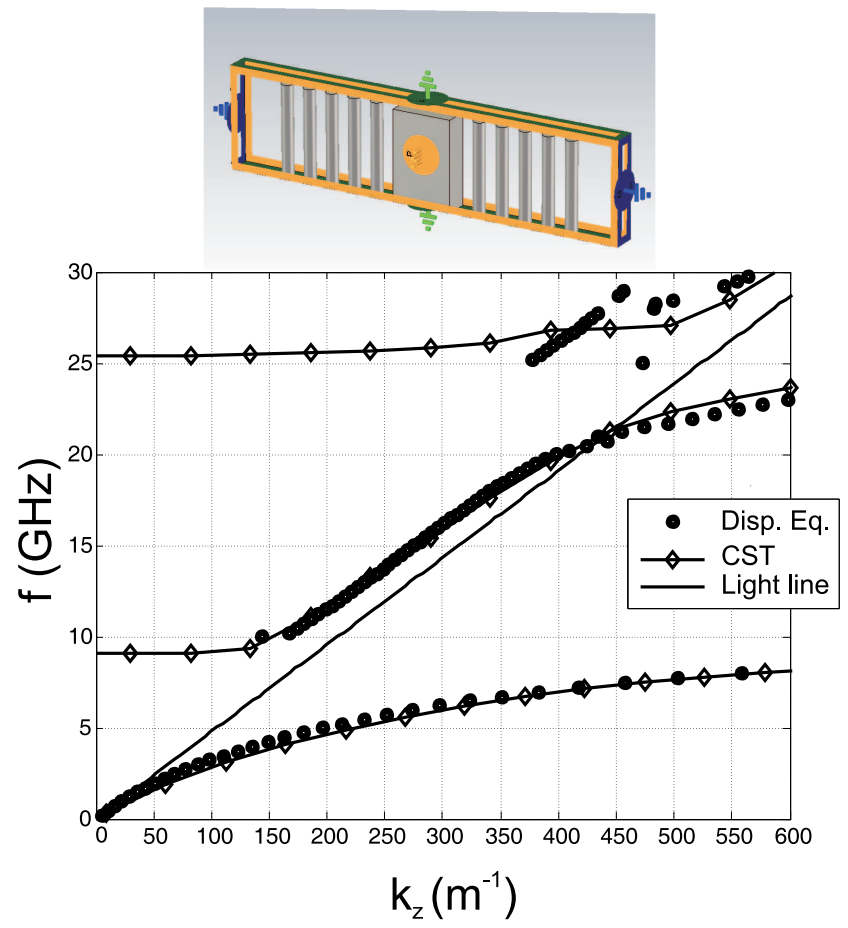

Fig. 8. Dispersion diagram for the ridge waveguide (dotted line). The geometry is the same as that reported in caption of Fig. 6, but including an inserted a $5 \mathrm{~mm}$ wide ridge. The dispersion diagram is compared with the one obtained through a CST Microwave Studio simulation (diamond line) of the reference structure inserted above the graph. The light line is also shown.

In the most interesting frequency band between 10 and $20 \mathrm{GHz}$, the approximations used work well, and the mode propagating along the ridge is really quasi-TEM, as expected. Once $k_{z}$ is found, all the remaining quantities in (16) can be determined as well. Notice that the CST eigenmode solver gives many more modes than the curves shown in Fig. 8 due to the equivalent boundary conditions set at $x= \pm w / 2$ (in the present case $w=5 \mathrm{~mm}$ ). This modes have been left out in Fig. 8 . Therefore, to avoid misunderstandings, we present the complete multimode dispersion diagram in Fig. 9 to give an idea about the multiplicity of modes that can be found in a practical packaging. We see that there are several $y$-attenuated modes below the lower bound of the bed of nails stopband. Other modes start appearing at $17 \mathrm{GHz}$. This first of these modes seems to have a cut-off within the parallel-plate stopband $10-17.5 \mathrm{GHz}$. The

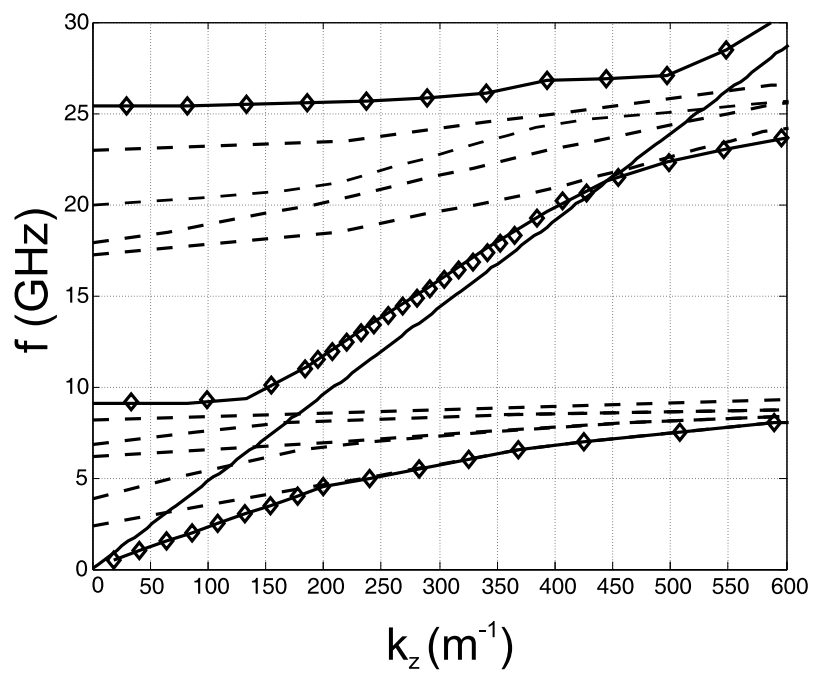

Fig. 9. Dispersion diagram obtained by CST Microwave Studio including all modes due to enclosure resonances. The diamond marked lines are the same as in Fig. 8. The light line is also shown.

apparently strange fact is readily explained: this mode is associated with an odd transverse variation along the ridge, which is large enough at that frequency. The cut-off frequency of this mode is actually the one which sets the upper bound of the unimodal region, for ridge width not so small. It is worth noticing that the stopband predicted in Fig. 6 for the parallel-plate structure without ridge still holds approximately when looking into the dispersion diagram with ridge in Fig. 8.

We stress the fact that in the lower frequency regime below the lower bound of the bed-of-nails band gap, the $x$-infinite structure supports a slow wave $\left(k_{z}>k\right)$. This means that in the transition region of the bed of nails, attenuation constants in (16a) and (16b) become propagation constants, and, as a consequence, the surface of nails cannot confine the field in the ridge region. This can be easily inferred from Fig. 7 .

In order to validate the modal field expansion approximation in (11), (13) and (15), the amplitude of the vertical $\left(E_{y}\right)$ electric field is plotted, in the same way as done in Fig. 7. Results are shown in Fig. 10. We have for simplicity drawn the field distribution inside the gap only, according to the assumption of homogenized surface. In Fig. 11, the normalized amplitude of $E_{y}$ and $H_{x}$ (in $\mathrm{dB}$ ) are compared with the same components obtained through CST, at $f=13 G H z$ (inside the band gap) and at a height $(y=h+d / 2)$, just in the middle of the gap region. The field distribution shows, as expected, a discontinuity at the region interfaces when the fields are evaluated in the middle of the gap. The reason for this is that the fields' continuity has been enforced only at the top cover. There, the results appear quite similar due to the small dimension of the gap, the only difference being the continuity of the approximated solution. However, the agreement between the numerical and analytical solutions looks reasonable, also in consideration of the following comments. Firstly, $E_{y}$ and $H_{x}$ from our approximated modal solutions show a different degree of decay. This is due to the fact that the decay of those components is associated with $\widetilde{\alpha}_{x}$ for $E_{y}$, and both $\widetilde{\alpha}_{x}$ and $\widetilde{\widetilde{\alpha}}_{x}$ for $H_{x}$. In particular, 

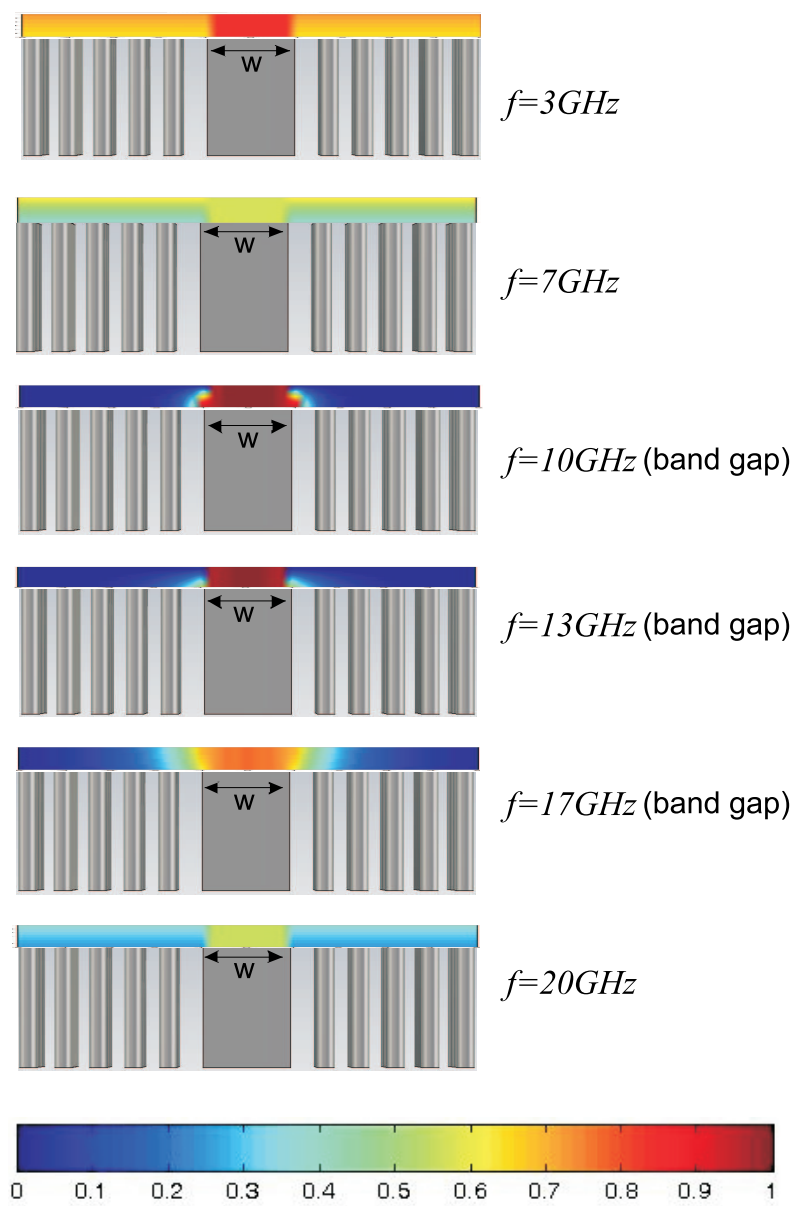

Fig. 10. Normalized amplitude of the vertical (along $y$ ) electric field for different frequencies calculated by the expressions in (11), (13) and (15)

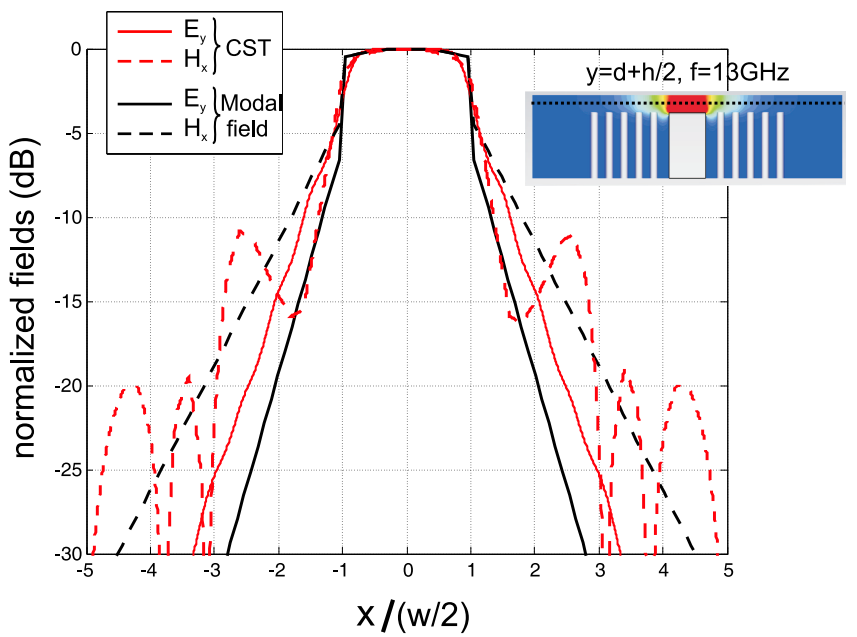

Fig. 11. Normalized amplitude (in $\mathrm{dB}$ ) of $E_{y}$ (solid line) and $H_{x}$ (dashed line) field distributions at $13 \mathrm{GHz}$, computed along the middle of the gap region $(y=d+h / 2)$ $\widetilde{\alpha}_{x}=\sqrt{k_{z}^{2}+\widetilde{k}_{y}^{2}-k^{2}}$ and $\widetilde{\widetilde{\alpha}}_{x}=\sqrt{k_{z}^{2}+\widetilde{\widetilde{k}}_{y}^{2}-k^{2}}$ are plotted in Fig. 12 in the stop band of the parallel-plate region, corresponding to the working bandwidth of the ridge gap waveguide. While $\widetilde{\alpha}_{x}$ is strongly varying with frequency and has a value greater than $100 \mathrm{~dB} / \lambda_{0}$ almost over the entire bandwidth, $\widetilde{\widetilde{\alpha}}_{x}$ is almost a constant in the same bandwidth. Indeed, since $k_{z} \simeq k$, $\widetilde{\widetilde{\alpha}}_{x} \rightarrow \widetilde{\widetilde{k}}_{y}=\pi /(h+d)$, and the resulting value is smaller over the bandwidth. This implies that $H_{x}$ component has a weaker decay, dictated by the attenuation factor $\widetilde{\widetilde{\alpha}}_{x}$. Secondly, regarding the CST $H_{x}$ component, we see oscillations in the nails region which are due to the actual periodicity of the pins, that we neglect in the surface impedance model. It is evident from Fig. 11 that the CST $E_{y}$ component does not show the same ripples. Indeed, $E_{y}$ has a stronger decay along $x$ than $H_{x}$, and this prevents $E_{y}$ from being influenced by the periodicity of the pins. The results are given in $d B / \lambda_{0}$, where $\lambda_{0}$ is calculated at

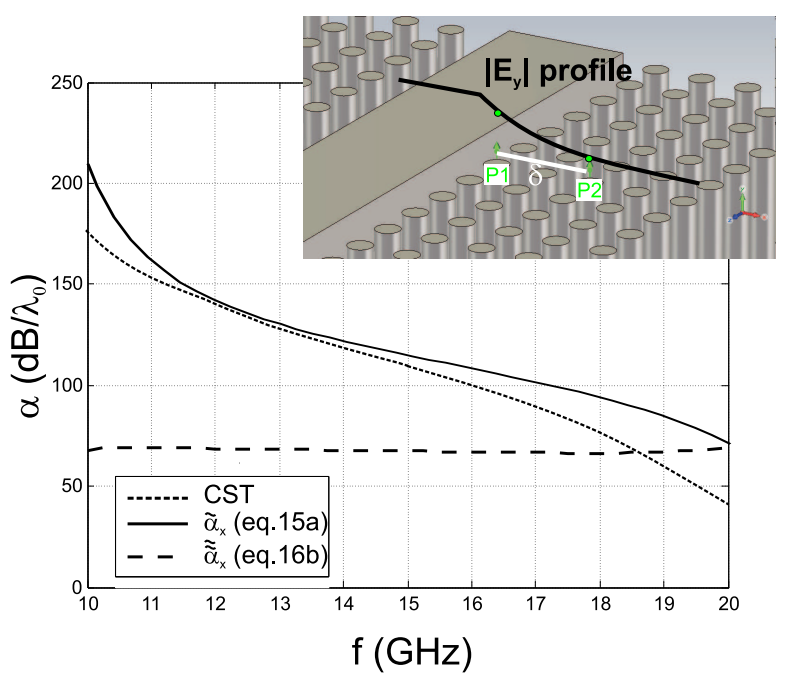

Fig. 12. Transverse attenuation constant in $x$-direction plotted as $d B / \lambda_{0}$ versus frequency compared with the one obtained through a CST simulation where the field decaying is captured by a couple of $\delta$-spaced probes located as shown in the inset.

$f_{0}=13 G H z$, the same frequency used in Fig. 11. The CST curve (dashed) in Fig. 12 has been obtained from the computed field values at a couple of field probes located at the wall of the smooth PEC plate, and spaced apart $\delta=2 a$ (see inset of Fig. 12). This procedure is valid in the limit where only one mode is present, and it seems to hold in our case. Indeed, results are in a good agreement, and they highlight that the attenuation has a value greater than $100 \mathrm{~d} B / \lambda_{0}$ almost over the entire bandwidth. The same result has not been generated for the $x$ component of the magnetic field, because of the strong ripple due to the periodicity of the pins.

\section{CONCLuSions}

We have presented an analytical solution for the fundamental quasi-TEM mode of a ridge gap waveguide where the parallelplate cut-off is realized using a bed of nails. The modal solution has been obtained by matching plane wave modal expressions in three regions; the region above the ridge and the two side regions 
above the bed of nails. The dispersion diagram so obtained well matches the one numerically predicted by a full-wave eigensolver and catches the main physical features of the structure, such as the rapid lateral field decay away from the ridge, showing values over $100 \mathrm{~dB}$ per wavelength within the stop band of the normal parallel-plate modes. The single-mode bandwidth of the ridge gap waveguide is mainly limited by the cut-off bandwidth of the surrounding bed of nails-type parallel-plate waveguide. This single mode bandwitdh is seen to be $10-17 \mathrm{GHz}$ in Fig.6, but it can also be larger if the gap size or ridge width are reduced. The cut-off bandwidth of parallel-plates with different metamaterial-type loadings of the lower surface are studied in [19], showing bandwidths up to two octaves. This has no relation to the surface band gap of the corresponding open surfaces, except for the lower cut-off frequency being approximately the same, which is clear by comparing results in [19] with results in [20]. The dispersion lines of the global parallel-plate modes, not included in Fig. 8, are then reported in Fig. 9, showing how they are affected by the enclosure having parallel-plate cut-off.

\section{REFERENCES}

[1] P.-S. Kildal, E. Alfonso, A. Valero-Nogueira, E. Rajo-Iglesias, "Local metamaterial-based waveguides in gaps between parallel metal plates," Antennas and Wireless Propagation letters (AWPL), Dec. 2008 [1]

[2] P.-S. Kildal, "Three Metamaterial-based Gap Waveguides between Parallel Metal Plates for mm/submm Waves," 3rd European Conference on Antennas and Propagation (EuCAP 2009), Berlin, Germany, 23-27 March 2009

[3] J. Hirokawa and M. Ando, "Single-layer feed waveguide consisting of posts for plane TEM wave excitation in parallel plates," IEEE Trans. Antennas Propag., vol. 46, no. 5, pp. 625-630, May 1998

[4] M.G. Silveirinha, C. A. Fernandes, J. R. Costa, "Electromagnetic Characterization of Textured Surfaces Formed by Metallic Pins," IEEE Trans. Antennas Propagat., vol. 56, n.2, pp. 405-415, Feb 2008

[5] P-S. Kildal, "Definition of artificially soft and hard surfaces for electromagnetic waves", Electronic Letters, Vol. 24, No. 3, pp. 168-170, 4th February 1988

[6] P.-S. Kildal and A. Kishk, "EM Modeling of surfaces with STOP or GO characteristics - artificial magnetic conductors and soft and hard surfaces", Applied Computational Electromagnetics Society Journal, Vol. 18, No. 1, pp. 32-40, March 2003

[7] A. Valero-Nogueira, E. Alfonso, J. I. Herranz, and M. Baquero, "Planar slot-array antenna fed by an oversized quasi-TEM waveguide," Microwave and Optical Technology Letters, vol. 49, pp. 1875-1877, Aug, 2007

[8] P. Padilla de la Torre, J.M. Fernndez and M. Sierra-Castaer, "Characterization of artificial magnetic conductor strips for parallel plate planar antennas", Microwave and Optical Technology Letters, vol. 50, n 2, pp. 498-504, Feb. 2008

[9] E. Alfonso, P.-S. Kildal, A. Valero, and J. I. Herranz, "Detection of local quasi-TEM waves in oversized waveguides with one hard wall for killing higher order global modes", IEEE International Symposium on Antennas and Propagation (IEEE AP-S), San Diego, July 2008

[10] M. Ng Mou Kehn and P.-S. Kildal, "Miniaturized rectangular hard waveguides for use in multi-frequency phased arrays", IEEE Transactions on Antennas and Propagation, vol. 53, no. 1, pp. 100-109, Jan 2005

[11] M. Ng Mou Kehn, M. Nannetti, A. Cucini, and S. Maci, P.-S. Kildal "Analysis of Dispersion in Dipole-FSS Loaded Hard Rectangular Waveguide", IEEE Transactions on Antennas and Propagation, vol. 54, no. 8, pp. 22752282, Aug 2006

[12] Z.Sipus, H. Merkel and P-S. Kildal, "Green's functions for planar soft and hard surfaces derived by asymptotic boundary conditions", IEE Proceedings Part H, Vol. 144, No. 5, pp. 321-328, Oct.1997

[13] P.A. Belov, R. Marques, S.I. Maslovski, I.S. Nefedov, M. Silveirinha, C. R. Simovski, and S. A. Tretyakov, "Strong spatial dispersion in wire media in the very large wavelength limit," Phys. Rev. B, vol. 67, pp. 113-103, Oct. 2003

[14] S.I. Maslovski, S.A. Tretyakov, and P.A. Belov, "Wire Media with Negative Effective Permittivity: a Quasi-stati Model", Microwave and Optical Technology Letters, vol. 35, No. 1, pp- 47-51, Oct. 2002

[15] R.J. King, D.V. Thiel, and K.S. Park, "The synthesis of surface reactance using an artificial dielectric," IEEE Trans. Antennas Propag., vol. 31, no. 3, p. 471, May 1983

[16] P.-S. Kildal, A. Kishk, Z.Sipus, "Asymptotic boundary conditions for strip-loaded and corrugated surfaces," Microwave and Optical Technology Letters, Vol. 14, pp. 99-101, Feb. 1997

[17] Z. Sipus, M. Bosiljevac, P.S. Kildal, "Local Wave Green's Functions of Parallel Plate Metamaterial-Based Gap Waveguide with One Hard Wall," IEEE International Symposium on Antennas and Propagation (IEEE AP. S), Charleston, June 2009

[18] www.mathworks.com

[19] E. Rajo-Iglesias, P.-S. Kildal, "Numerical studies of bandwidth of parallel plate cut-off realized by bed of nails, corrugations and mushroom-type EBG for use in gap waveguides", submitted to IET Microwaves, Antennas \& Propagation, May 2009

[20] E. Rajo Iglesias, M. Caiazzo, L. Inclan-Sanchez, and P.-S. Kildal, "Comparison of bandgaps of mushroom-type EBG surface and corrugated and strip-type soft surfaces", IET Microwaves, Antennas and Propagation, Vol. 1, No. 1, pp. 184-189, Feb 2007.

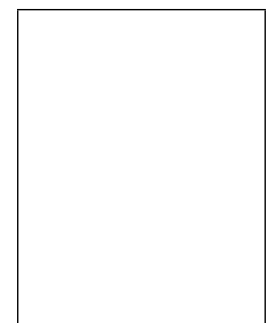

Alessia Polemi (S00, M04) was born in Casteldelpiano (Gr), Italy, on July 10, 1973. She received the Dr. Ing. degree in Engineering of Telecommunications (cum laude) from the University of Siena in July 1999. She received the Ph.D. degree in Information Engineering in March 2003. From January 2003 to October 2006 she was a post doc researcher at the same Department. Since November 2006 she has bees an Assistant Professor of Electromagnetic Fields at the Department of Information Engineering of the University of Modena and Reggio Emilia. Since November 2006 she has been the Italian Student Adviser for the Institution of Engineering and Technology (IET), and she is currently Associate Editor for the IET Italian Newsletter. In 2008, she was a visiting professor at the University of Pennsylvania, in Philadelphia, working on plasmonic structures. Her current research includes high frequency scattering theories, asymptotics electromagnetic methods, numerical electromagnetic methods, periodic structures, bandgap structures, antenna design, RFID systems, metamaterials and polaritons propagation at optical frequency.

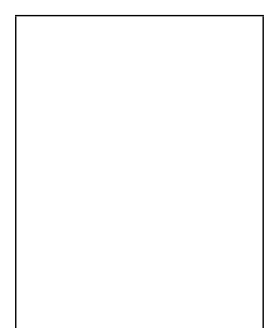

Stefano Maci Stefano Maci (S92, SM99, F04) was born in Rome in 1961. He received the Laurea degree (cum laude) in electronic engineering from the University of Florence, Italy, in 1987. Since 1998 he joined the University of Siena, Italy, where he presently is a Full Professor. He was a coauthor of an Incremental Theory of Diffraction for the description of a wide class of electromagnetic scattering phenomena at high frequency, and of a diffraction theory for the high frequency analysis of large truncated periodic structures. His research interests include: theory of Electromagnetism, integral equation methods, large phased array antennas, planar antennas and multilayer structures, reflector antennas and feed horns, metamaterials. He has been an invited speaker at many international conferences, and presenter of various short courses about EBG material, high-frequency methods, and computational methods. In 2004 he was founder, and he presently is the coordinator, of the European school of antennas (ESoA), a post graduate school comprising 26 courses and about 150 teachers from 20 European research centres. He was the responsible of several projects funded by the European Union (EU), by the European Space Agency (ESA-ESTEC) and by various European industries; he was Work Package leader in the Network of Excellence Antenna Center of Excellence (FP6, EU). He is principal author or coauthor of about 100 papers published in international journals, eight book Chapters, and about 300 papers in proceedings of international conferences. He was several times the recipient of best paper award in international journals and at conferences. Prof. Maci was an Associate Editor of the IEEE TRANSACTIONS ON EMC. He is currently an Associate Editor of the IEEE TRANSACTION ON ANTENNAS AND PROPAGATION and was twice Guest Editor of special issues of IEEE TRANSACTION ON ANTENNAS AND PROPAGATION. He was member of the Technical Advisory Board of the URSI Commission B, Convenor in a URSI General Assembly, member of the Delegate Assembly of the European Association of Antennas and Propagation, member of a NATO panel on metamaterials 
and MEMS, member of the scientific board of the Italian Society of Electromagnetism (SIEM), and coordinator of the board of the Italian Ph.D. school of Electromagnetism

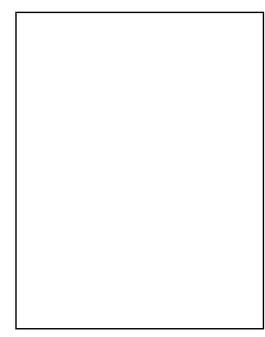

Per-Simon Kildal grew up and got his $\mathrm{PhD}$ in Norway, and he has been a Professor at Chalmers University of Technology, Gothenburg, Sweden since 1989. Kildal is Fellow of IEEE since 1995, and he has received two IEEE best journal article awards. His textbook "Foundations of Antennas" has been well received. Kildal has designed and analyzed two very large antennas using his own methods and computer codes: The EISCAT VHF parabolic cylinder, and the Gregorian dual-reflector feed of the Arecibo radiotelescope operated by Cornell University. He has three inventions related to antenna feeds: the hat feed (www.comhat.se), the dipoledisk feed with beam-forming ring, and the recent decade bandwidth "Eleven antenna" being a candidate for future wideband radio telescopes. The last ten years Kildal's research group has pioneered the development of the reverberation chamber into an accurate tool for OTA measurements of antennas and wireless terminals subject to Rayleigh fading (www.bluetest.se). Kildal is the originator of the concept of soft and hard surfaces in electromagnetics through which he and his coworkers preceded some of the recent research on electromagnetic bandgap surfaces and metamaterials. Recently this research resulted in a new local so-called ridge gap waveguide appearing in the gap between parallel metal plates, useful for applications above $30 \mathrm{GHz}$. 